\title{
On the History of the Michelson Experiment: A Personal Recollection (Gravitational Waves Detected 100 Years After Einstein's General Relativity)
}

\section{Haubold Hans $\mathrm{J}^{\star}$ and Haubold Barbara}

Centre for Mathematical and Statistical Sciences, Peechi Campus, KFRI, Kerala, India

"Corresponding author: Hans Haubold J, Centre for Mathematical and Statistical Sciences, Peechi Campus, KFRI, Peechi, Kerala, India, E-mail: hans.haubold@gmail.com

Received date: May 12, 2016; Accepted date: May 16, 2016; Published date: May 23, 2016

Copyright: (c) 2016 Haubold Hans J, et al. This is an open-access article distributed under the terms of the Creative Commons Attribution License, which permits unrestricted use, distribution, and reproduction in any medium, provided the original author and source are credited.

\section{Editorial}

For the first time, ripples in the fabric of space-time called gravitational waves, arriving at Earth from a cataclysmic event in the distant Universe, have been observed recently. This confirmed a major prediction of Albert Einstein's 1915 General Theory of Relativity and opened up an unprecedented new window to the Universe: gravitational wave astronomy [1] next to neutrino astronomy [2].

Gravitational waves carry information about their dramatic origins and about the nature of gravity that cannot otherwise be obtained. It has been concluded that the detected gravitational waves were produced during the final fraction of a second of the merger of two black holes to produce a single, more massive spinning black hole. Such a collision of two black holes had been predicted but never observed.

The gravitational waves were detected on September 14, 2015 at 5:53 a.m. Eastern Daylight Time (9:53 a.m. UTC) by both of the twin Laser Interferometer Gravitational-wave Observatory (LIGO) detectors, located in Livingston, Louisiana, and Hanford, Washington, USA [3].

Luminiferous aether was all the rage for physicists, for a very long time. Isaac Newton was the first to have thought up the idea of the existence of an all-pervading, invisible fluid, that he called aether. The aether engulfed the Universe. It was rarer inside dense materials, and denser in void. Newton used this magical medium as a crutch to lean on whenever his particle theory of light appeared to falter. As time passed by, Newton's particle theory of light eventually lost steam, especially when Thomas Young famously demonstrated the light's wave nature. Yet, the concept of aether persisted to haunt the physicist's minds. The wave theory of light had made the existence of the aether even more necessary as waves need a medium to travel through. Since light travels to Earth from distant stars and galaxies, the aether had to exist everywhere, in the intergalactic and the interstellar space as well. Besides being a transporter of light, the aether had to be invisible, mass-less, and at the same time extremely rigid. What was a desperate attempt by Newton to explain his faulty theory of light, had gradually turned into a difficult, self-contradicting and yet totally indispensable entity. In the middle of the 19th century, James Maxwell, who would reinforce the wave nature of light with his electromagnetic theory, suggested that the motion of the Earth through the aether must be detectable with optical experiments. And here, in 1881, entered the American experimenter Albert Abraham Michelson, who designed an exceptional experiment which elegantly laid the aether to rest, finally. It was the evidence for the non-existence of aether put forth by Michelson's interferometer experiments along with the boldness of Einstein who simply assumed that aether is not necessary for light to travel through, that laid the foundation for the revolutionary Special
Theory of Relativity. And history repeated 100 years later, when the model of Michelson's interferometer, utilized in LIGO, was the one that proved right a result of Einstein's General Theory of Relativity-the existence of the gravitational waves.

In what follows, we are recollecting information on our meetings with Dorothy Michelson Livingston (born 1 September 1906 in Chicago, died on 15 April 1994 in Wainscott, Long Island, NY) (Figure 1), the youngest daughter of "The Master of Light" and first American Nobel Prize Winner, Albert Abraham Michelson. These meetings concerned her writing of the biography of her father, the Einstein Centenary 1979 in Berlin, and the Michelson Anniversary 1981 in Potsdam.

Though Dorothy Michelson Livingston did not have a formal academic education she enrolled in several art schools and became an accomplished painter, specialized in water colors.

During WWII, Dorothy Michelson Livingston attended a welding school and worked in a factory producing war supplies. She was among those strong women replacing men who went off to war, particularly in metal and construction business. Together with the foreman she even founded her own company.

In the 1960s Dorothy Michelson Livingston began writing a very extensive and the only available biography of her father by corresponding with luminaries of physics around the world like J. Robert Oppenheimer, Max Born, and Louis de Broglie [4]. She could count on her memories meeting Albert Einstein when he met Albert A. Michelson.

Dorothy Michelson Livingston was invited by the Berlin Academy of Sciences to visit Potsdam in 1981, then belonging to East Germany, as the key guest of an International Colloquium in honor of the 100rd Anniversary of A.A. Michelson's ether-drift experiment. Back in the 1880s this experiment was an attempt to detect the relative motion of matter through the stationary luminiferous aether ("aether wind"). The result was negative, in that the expected difference between the speed of light in the direction of movement through the presumed aether, and the speed at right angles, was found not to exist. This result is generally considered to be the first strong evidence against the thenprevalent aether theory, and initiated a line of research that eventually led to Einstein's special relativity, which rules out a stationary aether (Figure 2). 
Citation: Hans HJ, Barbara H (2016) On the History of the Michelson Experiment: A Personal Recollection (Gravitational Waves Detected 100 Years After Einstein's General Relativity). Astrobiol Outreach 4: e116. doi:10.4172/2332-2519.1000e116

Page 2 of 4

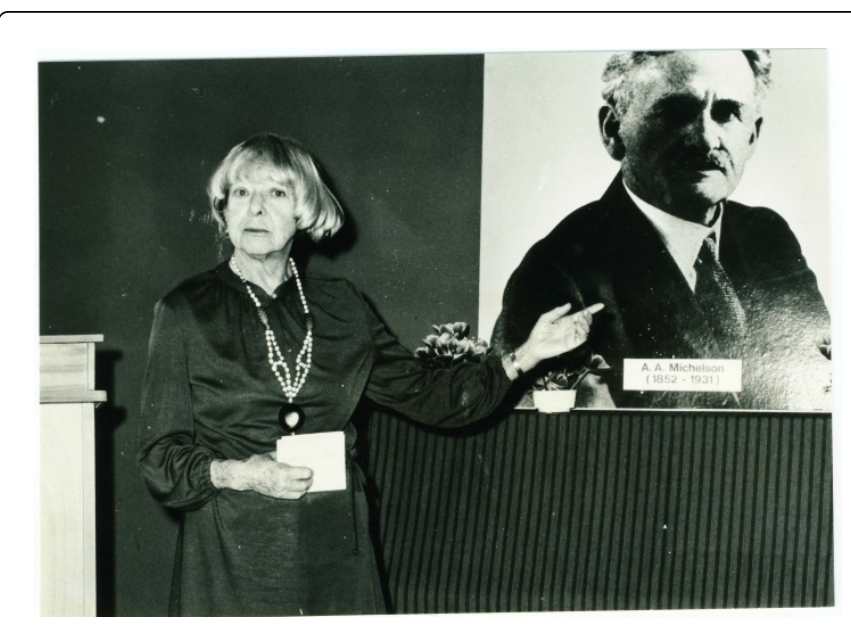

Figure 1: Dorothy Michelson Livingston (1981) opening the Michelson Colloquium in Potsdam, Germany, 27-30 April 1981.

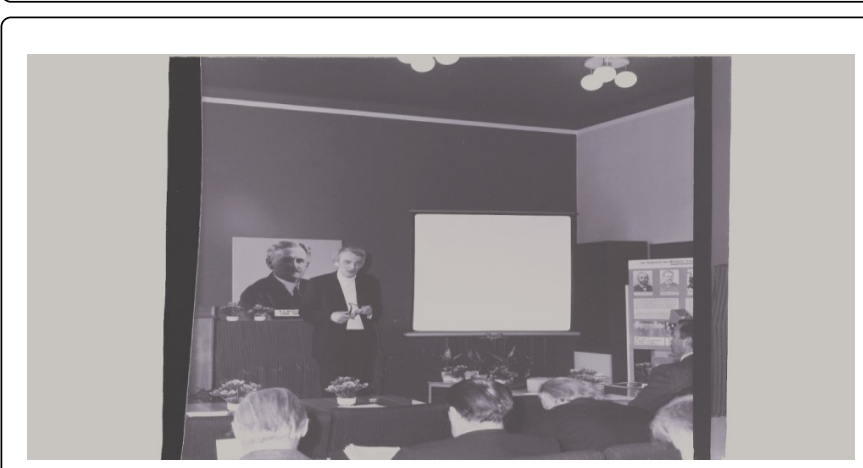

Figure 2: Prof. Dr. h.c. mult. Hans-Juergen Treder (born 4 September 1928 in Berlin; died 18 November 2006), Patron of the Michelson Colloquium at the opening ceremony of the Colloquium.

The Colloquium in Potsdam was officially hosted by the Astrophysikalisches Observatorium Potsdam (Astrophysical Observatory Potsdam, Figure 3) that comprises the Michelson cellar where Albert Abraham Michelson performed the experiment in 1881 for the first time that carries his name today (Figure 5) [9].

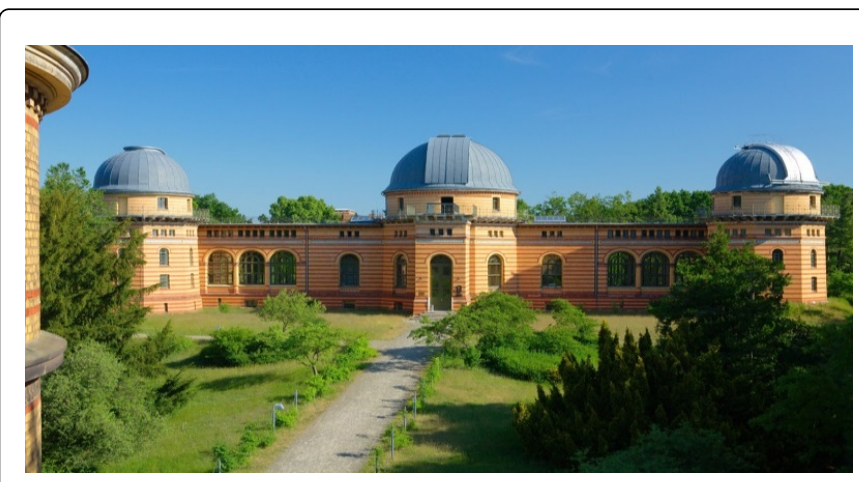

Figure 3: Astrophysical Observatory Potsdam, about 1979.
At the Colloquium, Dorothy Michelson Livingston certainly was the charismatic main guest and received the great honor to stay together with her daughter Ursula Ulmer (Switzerland) (Figure 4), who accompanied her on that visit, in the former summer house of Albert Einstein in Caputh (district of Potsdam). She was very curious and one may say positively surprised about the quality of the event, organized by the Zentralinstitut fuer Astrophysik der Akademie der Wissenschaften der DDR (Central Institute for Astrophysics of the Academy of Sciences of the German Democratic Republic).

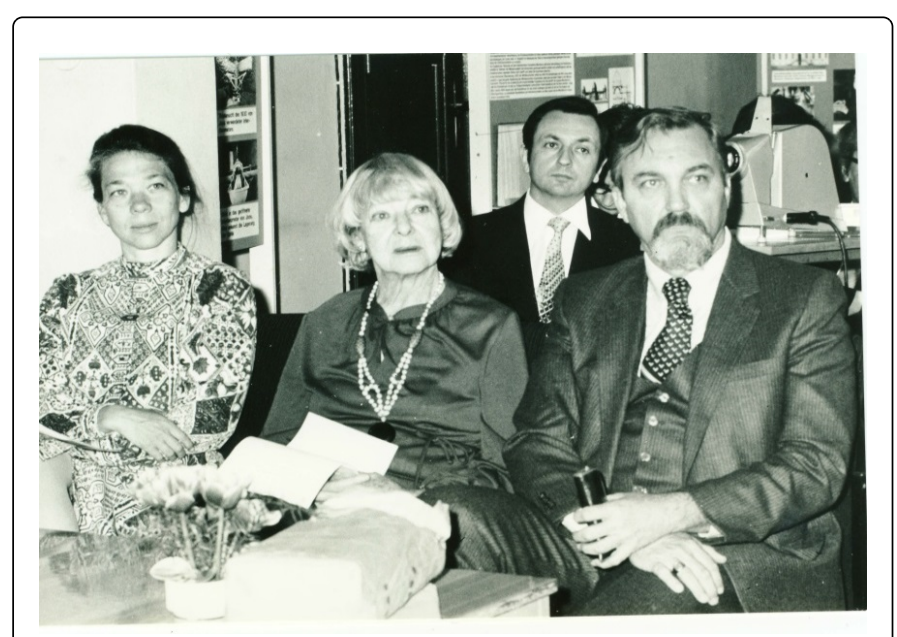

Figure 4: At the Michelson Colloquium in the City Hall of Babelsberg during the opening session from left to right U. Ulmer (youngest daughter of Dorothy Michelson Livingston), Dorothy Michelson Livingston, Rainer W. John, and L.S. Swenson).

In preparation of the Einstein Centenary, celebrated in Berlin in 1979 and the Michelson Anniversary, celebrated in Potsdam in 1981, young enthusiastic and dedicated staff scientists of the Observatory refurbished the cellular basement where Michelson placed his interferometer equipment (Figure 5) and set up an exhibition of scientific documents and photographs that reflected the situation of physics at the turn of the 19th century after Max Planck's discovery of black body radiation formula and before Alberts Einstein's Annus Mirabilis.

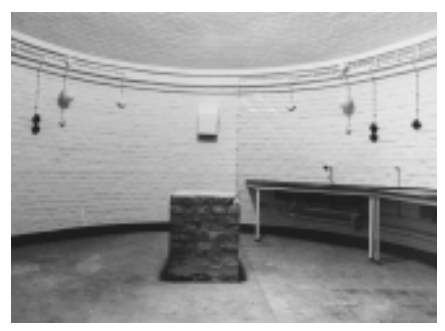

Figure 5: A.A. Michelson performed his experiment in this circular cellar (photo dated 1969) that is in the basement of the dome seen far right in Figure 3 (Haubold and John 1982).

The cellular basement was prepared and turned into a permanent exhibition for Einstein's Centenary in 1979 and served as the focal point for the exhibition at the time of the Michelson Colloquium in 1981. Many international renowned scientists were invited-not an easy 
Citation: Hans HJ, Barbara H (2016) On the History of the Michelson Experiment: A Personal Recollection (Gravitational Waves Detected 100 Years After Einstein's General Relativity). Astrobiol Outreach 4: e116. doi:10.4172/2332-2519.1000e116

Page 3 of 4

task taken into consideration the condition of "cold war". Hans J. Haubold and Rainer W. John, who were among the main organizers of the Colloquium accompanied Dorothy Michelson Livingston and also involved the author of this article in the organization of this unique event.

The Haubold family organized for Dorothy Michelson Livingstoon a trip from Potsdam and Caputh to Berlin in the small plastic car known as "Trabant" which was a great excitement for her. Also, Dody and Ursula were invited to the "Hans Otto Theater" for watching the performance of the opera "Hoffmann's Erzaehlungen" which they enjoyed tremendously. One Sunday morning-Dody and Ursula already had left Potsdam and Caputh and stayed in West-Berlin, the Haubolds received a phone call from them asking if we could meet in East-Berlin to visit the famous Pergamon Museum on the Museums Island. Pulling the pots from the stove where lunch was cooking, the Haubold family, including the three-year old son, hurried to Berlin. Together we strolled through the Museum, admiring the famous Pergamon Altar. Dody and Ursula were overwhelmed by the incredible treasure the Pergamon Museum had to offer.

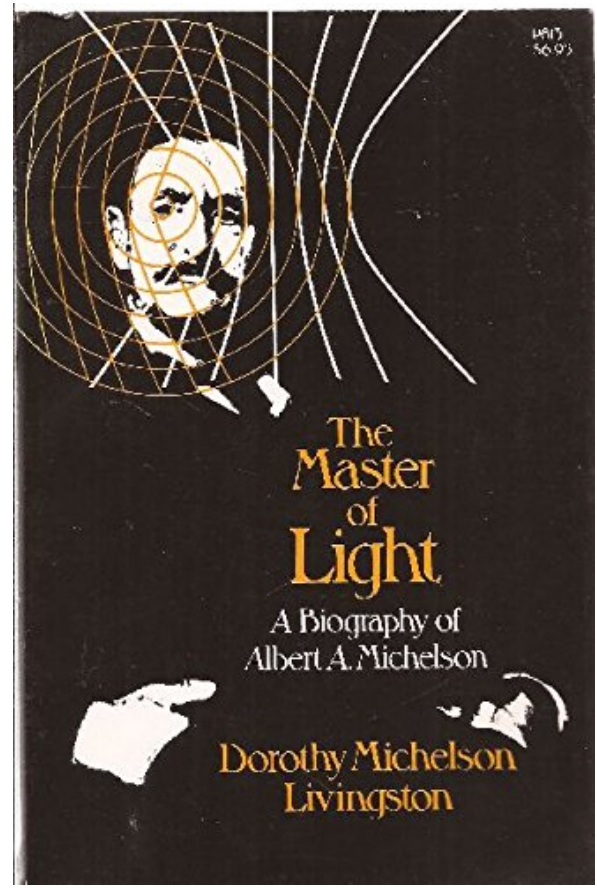

Figure 6: Cover of Dorothy Michelson Livingston's biography of her father (Michelson Livingston 1973).

And here we met Dody: An athletic, good looking lady with a gray bob, lively, curious, adventurous, interested and open minded to everything. No wonder that she, out of 6 siblings-three of Albert A. Michelson's first marriage and three of his second, where she was the youngest, had this drive and desire to learn what her famous father had achieved, how it was seen by the scientific community and beyond and how it was put into perspective to other results, mainly considered as milestones for Albert Einstein's theory of relativity (Haubold and Yasui 1986) [5]. Subsequent to the Michelson Colloquium in Potsdam in 1981, in 1987 the American Institute of Physics organized a grand international symposium titled Modern Physics in America: A Michelson-Morley Centennial Symposium, Cleveland, Ohio, USA,
October 30-31, that celebrated the famous greatly refined repetition of the original Michelson experiment in Potsdam that was performed at the Case Western Reserve University in Cleveland in 1887 [6].

Dorothy Michelson Livingston crowned her research with the monography "The Master of Light" (Figure 6).

In 1989-Haubolds had moved to New York since Hans J. Haubold was appointed to the Office for Outer Space Affairs of the United Nations-Dorothy Michelson Livingston and her husband Goodhue Livingston (Figure 7) (a former secretary to Mayor La Guardia [7]; among his ancestors was Robert R. Livingston) invited the Haubolds to their home at Wainscott, Long Island, New York.

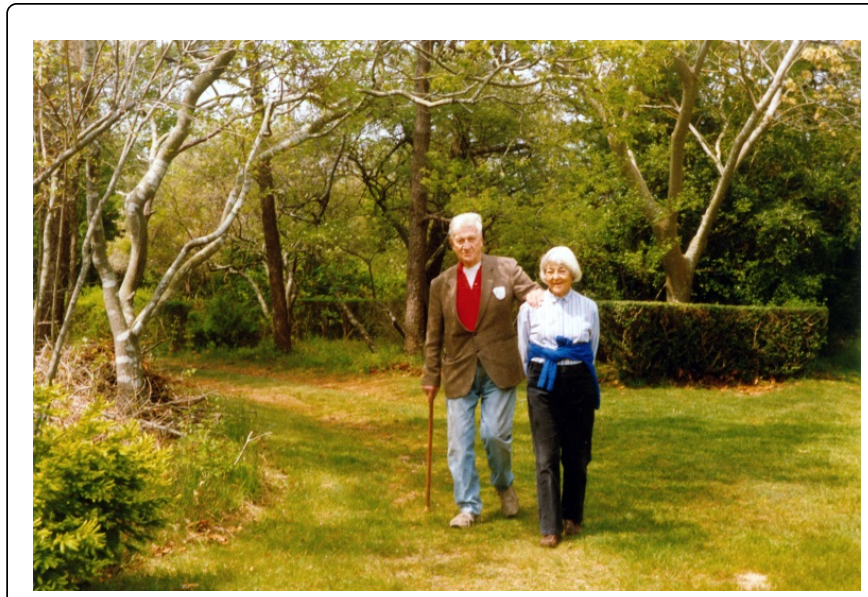

Figure 7: Goodhue Livingston and Dorothy Michelson Livingston walking at their Wainscott premises in 1991.

Excitedly, they accepted the invitation which was a great honor and spent a weekend with those two outstanding individuals and their dog, called Thatcher. During this time Dorothy Michelson Livingston provided Hans Haubold the opportunity to help sorting out and preparing the large collection of papers and documents which she possessed from her father. Haubolds spend a couple of weekends in the attic of the Garage to put things into order. This was very exciting as they came across of correspondence between A.A. Michelson and many famous scientists. Later, the well-sorted and archived documents were donated to the Nimitz Library of the United States Naval Academy at Annapolis [4].

The beautiful country house in Wainscott was an open spot where children, grandchildren and visitors were streaming in and out. In the cozy country-style living room, showing an impressive oil painted portrait of Goodhue Livingston, hundreds of books, journals and papers were scattered around and the whole environment breathed an atmosphere of intellect and true American self-conscience. At their cozy fireplace we listened to stories about the interesting life of the couple.

In 1994-the Haubolds had already returned to Vienna (Austria)-the couple died at age 88 (Dody) and 97 (Goodhue) [7]. This information was provided by Goodhue Livingston's well-known daughter Louisa Livingstone Kennedy [8]. They lay side by side on the Cemetery in Southhampton (Figure 8). 
Citation: Hans HJ, Barbara H (2016) On the History of the Michelson Experiment: A Personal Recollection (Gravitational Waves Detected 100 Years After Einstein's General Relativity). Astrobiol Outreach 4: e116. doi:10.4172/2332-2519.1000e116

Page 4 of 4

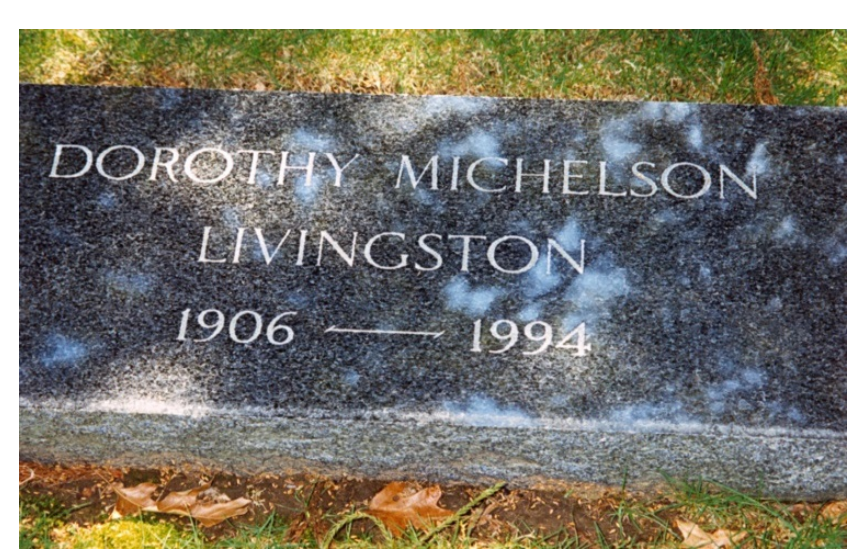

Figure 8: Grave stone of Dorothy Michelson Livingston at Southhampton Cemetary, New York.

\section{Selected Works by Dorothy Michelson-Livingston}

D. Michelson Livingston (1973) The Master of Light-A Biography of Albert A. Michelson, Charles Scribner's Sons, New York.

D. Michelson Livingston (1982) Einstein and Michelson-Artists in Science, Astronomische Nachrichten 303: 15-16.
D. Michelson Livingston (1988) Reminiscences of My Father, AIP Conference Proceedings 169: 22-24.

\section{References}

1. Blair D, Ju L, Zhao CN, Wen LQ, Chu QI, et al. (2016) Gravitational wave astronomy. arXiv.org > physics > arXiv:1602.02872

2. Ishihara A (2015) Neutrino astronomy. arXiv:1511.0382v1 [astro-ph.HE]

3. Castelvecchi D (2016) LIGOS's path to victory. Nature 530: 261-262.

4. Close Hubbard E (2003) A Guide to the Albert A. Michelson Collection 1803-1989, MS 347, A Collection in the Special Collections \& Archives Department, Nimitz Library, United States Naval Academy, 589 McNair Road, Annapolis, Maryland 21402-5029. Maryland: USA pp: 21402-5029.

5. Haubold HJ, Yasui E (1986) Jun Ishiwaras text ueber Albert Einsteins Gastvortrag an der Universitaet zu Kyoto am 14. December 1922. Archive for History of Exact Sciences 36: 271-279.

6. Haubold B, Haubold HJ, Pyenson L (1988) Michelson's First Ether-Drift Experiment in Berlin and Potsdam. AIP Conference Proceedings 179: $42-54$.

7. http://www.nytimes.com/1994/08/31/obituaries/goodhue-livingston-cityplanner-dies-at-97.html

8. http://www.nytimes.com/2007/08/28/us/28kennedy.html?_r=0

9. Haubold HJ, John RW (1982) Albert A. Michelsons AetherdriftExperiment 1880/1881 in Berlin und Potsdam. NTM-Schriftenreihe Geschichte der Naturwissenschaften, Technik und Medizin, Leipzig 19: 31-45. 